\title{
Förderung der Teilhabe am Arbeitsleben und der beruflichen (Re-)Integration bei Abhängigkeitserkrankungen aus Sicht des Fachverbandes Sucht e. V. (FVS)
}

V. Weissinger

\author{
Promoting Addictive Patients' Participation in Working Life and their \\ Professional (Re)integration from the Perspective of the Fachverband \\ Sucht e.V. (FVS; Addiction Related Association)
}

Im Aktionsplan Drogen und Sucht der Drogenbeauftragten der Bundesregierung aus dem Jahr 2003 wird festgestellt, dass Arbeitslosigkeit und die damit verbundenen Prozesse der Armutsentwicklung mit einer Vielzahl von psychosozialen Risiken verbunden sind, die verstärkend auf den Konsum von Suchtmitteln und die Entwicklung einer substanzbezogenen Abhängigkeit wirken können. Deshalb wird darauf hingewiesen, dass der Integration von suchtmittelabhängigen Menschen durch Arbeit und Beschäftigung eine sehr große Bedeutung zukommt. Zur Teilhabe am Arbeitsleben müssten verbindliche Vereinbarungen mit den Trägern der medizinischen Rehabilitation über die Kooperation in der Rehabilitation, aber auch mit den zuständigen Arbeitsverwaltungen getroffen werden, so eine wesentliche Forderung aus dem Aktionsplan Drogen und Sucht.

\section{„Wolfsburger Resolution“ und weitere Umsetzungsschritte}

Vor diesem Hintergrund entstand die Initiative des Fachverbandes Sucht e.V., die zur „Wolfsburger Resolution zur Förderung der Teilhabe am Arbeitsleben von suchtkranken Menschen vom 4.12.2003“ führte [1]. Diese wurde mit breiter Zustimmung unterschiedlicher Organisationen, wie Rentenversicherungsträgern, Krankenkassen, Bundesagentur für Arbeit, Unfallversicherung, Bundesarbeitsgemeinschaft der Integrationsämter sowie der Leistungserbringer und des Beauftragten für die Belange behinderter Menschen verabschiedet.

In dieser Resolution wird hervorgehoben, dass die Teilhabe am $\mathrm{Ar}$ beitsleben einen entscheidenden Einflussfaktor zur Aufrechterhaltung von Suchtmittelabstinenz und damit zur Stabilisierung des gesundheitlichen Zustandes des Rehabilitanden nach einer erfolgten medizinischen Rehabilitation darstellt. Von daher sollten die Anstrengungen verstärkt werden, den Behandlungserfolg durch das passgenaue, nahtlose und zügige Zusammenwirken der medizinischen Rehabilitationsleistungen mit den spezifischen Leistungsangeboten zur beruflichen Wiedereingliederung zu festigen. Hierzu zählen:

- ein prozessorientiertes Rehabilitations- und Eingliederungsmanagement und damit verbunden eine nahtlose Leistungserbringung;

- der Einsatz arbeitsbezogener Maßnahmen während der Entwöhnungsbehandlung unter frühzeitigem Einbezug der Leistungsträger bzw. deren Rehabilitationsberater;

- spezifische Angebote für Abhängigkeitskranke mit besonderen Teilhabeproblemen in Form von Leistungseinschränkungen.

Von besonderer Bedeutung hinsichtlich der Verbesserung von Schnittstellenproblemen sind aus Sicht der medizinischen Rehabilitationseinrichtungen hierbei der frühzeitige Einbezug der zuständigen Leistungsträger zur Förderung der Teilhabe am Arbeitsleben. So ist bereits während einer medizinischen Rehabilitationsmaßnahme in Zusammenarbeit mit den zuständigen Stellen und dem Rehabilitanden zu prüfen, ob weiterführende Leistungen zur Förderung der Teilhabe am Arbeitsleben erforderlich sind.

Übergänge zwischen den verschiedenen Leistungen (z.B. zwischen medizinischen und beruflichen Rehabilitationsleistungen, medizinischen Rehabilitationsleistungen und Qualifikationsbzw. Vermittlungsleistungen der Bundesagentur für Arbeit/anderen Leistungsträgern) sollten möglichst nahtlos gestaltet werden, um die beruflichen (Re-) Integrationschancen zu erhöhen.

Institutsangaben

Fachverband Sucht e. V., Bonn

Korrespondenzadiresse

Dr. Volker Weissinger · Fachverband Sucht e.V. · Walramstraße 3 · 53175 Bonn ·E-mail: sucht@sucht.de .

www.sucht.de

Bibliografie

Suchttherapie 2005; 6: 182 - 185 @ Georg Thieme Verlag KG Stuttgart · New York

DOI 10.1055/s-2005-858809

ISSN 1439-9903 
Die Förderung der Teilhabe am Erwerbsleben ist im Indikationsbereich Abhängigkeitserkrankungen von zentraler Bedeutung, da ein hoher Anteil von Patienten/-innen arbeitslos ist (Basisdokumentation des FVS: stationäre Rehabilitation Alkohol-/Medikamentenabhängiger, Entlassjahrgang 2003: Anteil der Arbeitslosen: 39,1\%) [2].

Einrichtungen in den neuen Bundesländern mit einem besonders hohen Anteil Erwerbsloser und schwierigen strukturellen Rahmenbedingungen sind vor besondere Herausforderungen gestellt.

Problematisch ist, dass sich die Agenturen für Arbeit und Arbeitsgemeinschaften häufig erst dann als zuständig ansehen, wenn die Wiederherstellung der Erwerbsfähigkeit gewährleistet ist und die Patienten dem Arbeitsmarkt wieder zur Verfügung stehen.

In der Folge der Wolfsburger Resolution fand am 1.6.2004 ein Gespräch zwischen Vertretern des VDR (Verband Deutscher Rentenversicherungsträger), der BfA (Bundesversicherungsanstalt für Angestellte) und der BA (Bundesagentur für Arbeit) auf Initiative des FVS statt. Im Rahmen dieses Gespräches wurden verschiedene Problembereiche der beruflichen Wiedereingliederung von Suchtkranken angesprochen. Zentrale Aspekte dieses Gesprächs waren, dass

- regionale Ansprechpartner in den Agenturen für Arbeit für suchtkranke Menschen zur Verfügung stehen sollen und

- bereits während der medizinischen Rehabilitationsleistung Kontakt mit den Agenturen für Arbeit aufgenommen werden kann, um Perspektiven für die Teilhabe am Arbeitsleben zu entwickeln und entsprechende Schritte frühzeitig einzuleiten.

In der Folge dieses Gespräches wurde eine Handlungsempfehlung/Geschäftsanweisung der BA am 20.11.2004 herausgegeben, welche regelt,

- dass bei der beruflichen Integration von Rehabilitanden in der medizinischen Rehabilitation (insbesondere Entwöhnungsbehandlung) das erste Beratungsgespräch in den Agenturen für Arbeit von dem für Reha-/SB zuständigen Team auf Beraterebene (Anlaufstelle für die Ratsuchenden bzw. Sozialdienste der Fachkliniken) vorbereitet wird. In diesem Zusammenhang wird auch festgelegt, ob die weitere Betreuung im Team verbleibt oder durch ein anderes Team (Vermittlungsstelle/ Beratung) erfolgt;

- dass bereits während der medizinischen Maßnahme den Suchtkranken die Möglichkeit einer Familienheimfahrt eingeräumt wird und dem bei der Terminierung des Beratungsgesprächs Rechnung getragen werden soll (Tab.1).

Ferner wurde infolge der oben genannten Gespräche und Aktivitäten der Leitfaden für die ärztliche Begutachtung der BA „Missbrauch und Abhängigkeit von Drogen und Medikamenten" überarbeitet (Stand: November 2004). Dies geschah vor dem Hintergrund, dass Katamnesestudien zeigen, dass die Rückfallhäufigkeit bei arbeitslosen Patienten/-innen nach einer stationären Entwöhnungsbehandlung signifikant erhöht ist und die meisten Rückfälle nach einer stationären Rehabilitationsmaßnahme bei Alkoholabhängigkeit in den ersten Monaten nach der Behandlung erfolgen. So ergab die klinikübergreifende Studie des FVS [3] des Entlassjahrgangs 2003 bei alkohol-, medikamentenabhängi-
Tab. 1 Handlungsempfehlung/

Geschäftsanweisung der BA, Originaltext

Geschäftszeichen PP51-6530

20. November 2004

Beratung von Rehabilitanden in der medizinischen Rehabilitation (insbesondere Entwöhnungsbehandlung)

1. Frühzeitige Kontaktaufnahme mit der örtlich zuständigen Agentur

Sowohl aus medizinischer Sicht (Stabilisierung des Therapieerfolges) wie auch aus BA-Sicht (frühzeitige Beratung und Einleitung von Vermittlungsbemühungen) soll noch während der medizinischen Maßnahme eine Kontaktaufnahme mit der örtlich zuständigen Agentur erfolgen. Ziel ist, die Integration in Arbeit einschließlich evtl. notwendiger Leistungen zur Teilhabe am Arbeitsleben möglichst nahtlos zu gestalten.

Um dem besonderen Unterstützungsbedarf dieses Personenkreises bei der beruflichen Integration Rechnung zu tragen, soll das erste Beratungsgespräch in den Agenturen von dem für Reha/SB zuständigen Team auf Beraterebene (Anlaufstelle für die Ratsuchenden bzw. Sozialdienste der Fachkliniken) vorbereitet werden. In diesem Zusammenhang wird auch

festgelegt, ob die weitere Betreuung im Team verbleibt oder durch ein anderes Team (Vermittlung/Beratung) erfolgt.

2. Terminiertes Beratungsgespräch

Während der medizinischen Maßnahme wird den Suchtkranken in der Rege die Möglichkeit einer Familienheimfahrt eingeräumt. Dem soll bei der Terminierung des Beratungsgesprächs Rechnung getragen werden.

gen Patienten, dass von 773 rückfälligen Kantamneseantwortern 54,1\% im Zeitraum von bis zu 3 Monaten und 79,9\% von bis zu 6 Monaten nach erfolgter Behandlung rückfällig geworden sind.

Dies verdeutlicht, dass zur Stabilisierung der Abstinenz neben weiteren Maßnahmen (z.B. Nachsorge, Teilnahme an Selbsthilfegruppen) auch der direkte Anschluss entsprechender Leistungen zur Teilhabe am Arbeitsleben nach erfolgter Entwöhnungsbehandlung beitragen kann. Dem trägt die Klarstellung, die im überarbeiteten Leitfaden (Version 2004, S. 15) [4] erfolgt, Rechnung. Diese enthält im Vergleich zur Vorgängerversion (November 2002) den Passus wie in Tab. 2.

Um einen Überblick über die derzeitige Einschätzung der aktuellen Tendenzen zur beruflichen (Re-) Integration in den ambulanten und stationären Behandlungseinrichtungen $\mathrm{zu}$ erhalten, entwickelten die Suchtverbände Deutsche Hauptstelle für Suchtfragen (DHS), Fachverband Drogen und Rauschmittel (FDR) und Fachverband Sucht e.V. (FVS) einen Fragebogen [5], der an die Mitgliedseinrichtungen verschickt wurde. Nachfolgend sind ausschließlich Ergebnisse der stationären Rehabilitationseinrichtungen (Fachkliniken für Alkohol/Medikamente und Drogen, Adaptionseinrichtungen) des Fachverbandes Sucht e.V. wiedergegeben. Von 56 angeschriebenen Einrichtungen antworteten 33, so dass ein Rücklauf von $58,9 \%$ vorliegt. Die nachfolgenden Angaben beziehen sich auf die Antworter, unter denen sich 21 Fachkliniken für Alkohol/Medikamente, 6 Fachkliniken für Drogen und 6 Adaptionseinrichtungen befanden. Der Anteil Arbeitsloser im Jahr 2004 betrug bei allen Fachkliniken für Alkohol/Medikamente 47,2\%, deutlich höhere Anteile wiesen hierbei die Einrichtungen in den neuen Bundesländern aus (63,0\%). Bei den Fachkliniken für Drogen und Adaptionseinrichtungen lagen die Anteile Arbeitsloser noch höher (83,5 bzw. $89,9 \%)$.

Von den Ergebnissen, die zu einem späteren Zeitpunkt publiziert werden, sollen an dieser Stelle nur solche berichtet werden, die 
sich auf die Bewertung der Kooperation mit den jeweiligen Partnern beziehen. Tab. 3, die nach Anzahl der Nennungen geordnet ist, gibt hierzu einen Überblick über die Bewertung der antwortenden Einrichtungen.

Überwiegend positiv wurde die Zusammenarbeit mit den Betrieben im Rahmen der externen Arbeitserprobung, den Adaptionseinrichtungen und den Fachberatern der Rentenversicherung bewertet. Die Zusammenarbeit mit den Arbeitsvermittlern/Fachberatern der Agenturen für Arbeit/Arbeitsgemeinschaften wurde von 68,8\%

Tab. 2 Leitfaden für die arbeitsamtsärztliche Begutachtung Missbrauch und Abhängigkeit von Alkohol, Drogen und Medikamenten.

\section{Version 2002}

"Schulungsmaßnahmen setzen Abstinenz voraus. Sie stellen unterschiedlich hohe Anforderungen an die körperliche, geistige und psychische Belastbarkeit. Es sollte individuell und unter Berücksichtigung der Besonderheit der jeweils vorgesehenen beruflichen Reha-Maßnahme beurteilt werden, welche Abstinenzdauer vor Befürwortung einer Schulungsmaßnahme nachzuweisen ist. Bei längerfristigen Leistungen zur Teilhabe am Arbeitsleben, die z.B. in einem neuen Berufsabschluss münden, ist i.d.R. wegen der damit verbundenen besonderen Anforderungen und erst nach längerer Zeit zunehmend sicher abschätzbarer Prognose eine Abstinenzdauer von mindestens 6 , nach Einzelfall auch 12 Monaten vorauszusetzen."

\section{neue Version 2004}

„Schulungsmaßnahmen setzen Suchtmittelabstinenz voraus. Andererseits spielt der frühzeitige Einsatz von Maßnahmen zur Teilhabe am Arbeitsleben eine nicht zu unterschätzende Rolle für die Stabilisierung der Abstinenz. Der Einsatz dieser Maßnahmen setzt maßnahmebegleitend regelmäßige Abstinenzkontrollen und eine wiederholte Überprüfung einer Prognoseeinschätzung voraus.

Grundsätzlich sollten modulare berufsfeldnahe Maßnahmen (Teilqualifikation, berufsvorbereitende Lehrgänge, Betriebspraktika etc.) im Vordergrund der Überlegung stehen. Längerfristige Maßnahmen kommen in der Regel erst nach einer Stabilisierung des Therapieerfolges mit 6-12-monatiger Abstinenz in Betracht.

Ein schematisches Abwarten der Prüfung und Einleitung von Leistungen zur Teilhabe am Arbeitsleben bzw. eine dementsprechende gutachterliche Empfehlung ist aber fachlich nicht gerechtfertigt. In gut zu begründenden Fällen mit günstiger Prognose können deshalb auch längerfristige Maßnahmen, z. B. eine Umschulung, schon frühzeitig nach einer Entwöhnungsbehandlung eingeleitet werden.“ als befriedigend und von $21,9 \%$ als mangelhaft/ungenügend eingestuft. Lediglich 9,4\% befanden diese als sehr gut/gut. Diese Ergebnisse sind vor dem Hintergrund zu sehen, dass zum Zeitpunkt der Befragung in vielen Städten und Kommunen infolge der gesetzlich vorgegebenen neuen Zuständigkeitsregelungen Umstrukturierungsprozesse im Gange waren. Gering ausgeprägt ist die Zusammenarbeit mit privaten Arbeitsvermittlern, Arbeitsloseninitiativen, Trägern öffentlich geförderter Arbeitsgelegenheiten oder beruflichen Trainingszentren. Auch mit Berufsförderungswerken und Berufsbildungswerken arbeitet nur ein Teil der Einrichtungen zusammen. 90,9\% der befragten Einrichtungen sind der Meinung, dass die bestehenden Angebote zur Förderung der Teilhabe am Arbeitsleben nicht ausreichen.

\section{Weitere Erfordernisse und Schlussfolgerungen}

Es zeigt sich, dass hinsichtlich der Umsetzung der Wolfsburger Resolution und der Handlungsempfehlung der BA vom 20.11.2004 noch erheblicher Handlungsbedarf besteht. Zusammenfassend lässt sich für die Zukunft auch aufgrund der oben genannten Befragung von Mitgliedseinrichtungen fordern, dass folgende Aspekte bereits während der medizinischen Rehabilitationsphase Berücksichtigung finden sollten:

- regelmäßige Präsenz der Arbeitsvermittler und Reha-Berater der Leistungsträger in der stationären Rehabilitationseinrichtung (individuelles Case-Management) bzw. qualifizierte Beratung durch die Agenturen für Arbeit, Arbeitsgemeinschaften und Jobcenter am Wohnort während der Entwöhnungsbehandlung;

- Mitbeurteilung der Reintegrationsmöglichkeiten in den $\mathrm{Ar}$ beitsmarkt sowie des Qualifizierungsbedarfs und der -möglichkeiten sowie der geeigneten Förderinstrumente durch die Reha-Berater der Leistungsträger;

- Entwicklung von Perspektiven, falls eine Wiedereingliederung ins Erwerbsleben hochgradig gefährdet bzw. nicht möglich erscheint;

- frühzeitige Bewilligung der Adaptionsphase (Phase II der medizinischen Rehabilitation) bei Vorliegen der entsprechenden Voraussetzungen;

- verbindliche Übernahme der Kosten für die Adaptionsbehandlung durch die Krankenkassen bei entsprechender Zuständigkeit;

\section{Kooperationspartner}

\section{Bewertung der Kooperationspartner sehrgut/gut befriedigend mangelhaft/ $n$ ungenügend}

\begin{tabular}{|c|c|c|c|c|}
\hline $\begin{array}{l}\text { Arbeitsvermittler/Fachberater der Agenturen } \\
\text { für Arbeit, kommunale Arbeitsgemeinschaften }\end{array}$ & $9,4(3)$ & $68,8(22)$ & $21,9(7)$ & 32 \\
\hline Fachberater der Rentenversicherung & $80,6(25)$ & $19,4(6)$ & $0,0(0)$ & 31 \\
\hline externe Arbeitserprobung & $100,0(31)$ & $0,0(0)$ & $0,0(0)$ & 31 \\
\hline Adaptionseinrichtungen & $96,4(27)$ & $0,0(0)$ & $3,6(1)$ & 28 \\
\hline berufliche Trainingszentren & $50,0(7)$ & $42,9(6)$ & $7,1(1)$ & 14 \\
\hline Berufsförderungswerke, Berufsbildungswerke & $36,8(7)$ & $47,4(9)$ & $15,8(3)$ & 19 \\
\hline $\begin{array}{l}\text { Träger von Maßnahmen gemäß § } 16 \text { (3) SGB ॥I } \\
\text { (öffentlich geförderte Arbeitsgelegenheiten) }\end{array}$ & $12,5(1)$ & $50,0(4)$ & $37,5(3)$ & 8 \\
\hline Arbeitsloseninitiativen & $28,6(2)$ & $28,6(2)$ & $42,8(3)$ & 7 \\
\hline private Arbeitsvermittler & $0,0(0)$ & $80,0(4)$ & $20,0(1)$ & 5 \\
\hline
\end{tabular}

Tab. 3 Ergebnisse zur Frage: Mit welchen Kooperationspartnern arbeiten Sie auf dem Arbeitsfeld „Integration in Beschäftigung“ zusammen und wie bewerten Sie die Zusammenarbeit? Angaben in \%, $\mathrm{n}$ in Klammern 
- frühzeitige Zuleitung der entsprechenden Antragsunterlagen (mit Einverständnis des Versicherten) an die zuständigen Stellen des Arbeitsamtes bzw. der Rentenversicherungsträger bereits während der Rehabilitationsphase;

- Bewilligung von Qualifizierungsmaßnahmen bzw. von Maßnahmen zur Förderung der beruflichen Wiedereingliederung bereits während der medizinischen Rehabilitationsmaßnahme;

- Einschalten von privaten Arbeitsvermittlern bereits während der medizinischen Rehabilitation.

Positiv ist zu vermerken, dass beispielsweise die BfA die frühzeitige Kontaktaufnahme mit den Agenturen für Arbeit bereits während der Leistungen zur medizinischen Rehabilitation unterstützt (Rundschreiben vom 13.5.2005) [6]. So soll die Terminierung eines Beratungsgespräches rechtzeitig während der Leistungen zur medizinischen Rehabilitation erfolgen. Dieser Termin soll mit dem Rehabilitanden vorbereitet und er soll bei der Zusammenstellung der erforderlichen Unterlagen unterstützt werden. Auch für Rehabilitanden, die aufgrund der Dauer des voraussichtlichen Aufenthalts in der Rehabilitationseinrichtung keinen Anspruch auf Reisekostenerstattung bei Familienheimfahrten hätten, werden die Fahrtkosten zu einem Beratungsgespräch bei der örtlich zuständigen Agentur für Arbeit durch die BfA übernommen. Ferner ist die Inanspruchnahmemöglichkeit von erforderlichen Qualifizierungsangeboten für alkoholkranke Menschen nach erfolgter Rehabilitation zu gewährleisten.

Es bleibt zu befürchten, dass eine Förderung von Nichtleistungsbeziehern und Arbeitslosengeld-II-Beziehern in Form von Qualifizierungsmaßnahmen oder Umschulungen zukünftig zunehmend schwieriger wird, zumal auch die Anforderungen an die Zumutbarkeit der Arbeit im SGB II deutlich verschärft wurden.

Auch bleibt angesichts der vergleichsweise hohen Kosten, die Arbeitslose vor dem Bezug von Arbeitslosengeld II verursachen, abzuwarten, inwieweit sich die Aktivitäten der Agenturen für Arbeit und Personal-Service-Agenturen hinsichtlich Beratung, Vermittlung und Qualifizierung vorrangig auf diesen Personenkreis konzentrieren, um insbesondere deren Langzeitarbeitslosigkeit abzuwenden. Damit könnte die Gruppe der schwer vermittelbaren Arbeitslosen eine zusätzliche und erhebliche Benachteiligung erfahren, wodurch deren Chancen auf eine berufliche Wiedereingliederung erheblich eingeschränkt würden. Grundsätzlich ist es von entscheidender Bedeutung, dass feste Ansprechpartner für Suchterkrankungen in den Agenturen für Arbeit, Jobcentern und Sozialämtern etabliert werden, die Klienten/-innen wie Einrichtung informieren, beraten und behördenintern die besonderen Belange der Suchtkranken kennen und einbringen.

Dringend erforderlich sind insbesondere auch klare Regelungen und Zuständigkeiten infolge der Umsetzung der Hartz-Gesetze. Dies betrifft folgende Fragen:

- Wer ist bei einer Gesamtdauer der Rehabilitation von über 6 Monaten zuständig?

- Ist die jeweilige Behörde bei ALG-II- bzw. Sozialhilfeempfängern am Wohn- oder Behandlungsort zuständig?
Auch hinsichtlich der Höhe des Arbeitslosengeldes II gibt es unterschiedliche Verfahrensweisen der jeweils zuständigen Stellen (z.B. Abzüge von Verpflegungspauschalen). Vermieden werden muss, dass infolge ungeklärter Zuständigkeiten Patienten/-innen zunehmend verunsichert werden, ihre existenzielle Grundlage ungeklärt bleibt und es deshalb gehäuft zu Behandlungsabbrüchen kommt oder die Nichtantrittsquote einer Rehabilitationsleistung steigt.

\section{Ausblick}

Abschließend sei darauf verwiesen, dass zur Begleitung der weiteren Umsetzung des Aktionsplans Drogen und Sucht von der Bundesregierung der Drogen- und Suchtrat ins Leben gerufen wurde. Dieser hat verschiedene Arbeitsgruppen eingesetzt, davon eine zum Thema „Schnittstellenprobleme“. In dieser Arbeitsgruppe, an der Vertreter der relevanten Organisationen teilnehmen, wurde u.a. die Frage der Umsetzung der Handlungsempfehlung der BA vom 20.11.2004 diskutiert. Ergebnis der Besprechung war, dass der VDR (Verband Deutscher Rentenversicherungsträger) die Problematik noch einmal offiziell an die Bundesagentur für Arbeit herantragen wird. Die Bundesagentur wird dann etwaigen Änderungsbedarf in ihrer Handlungsempfehlung/Geschäftsanweisung vom 20.11.2004 überprüfen und ihre Umsetzung optimieren. Auch hält die Arbeitsgruppe eine frühzeitige Einleitung beruflicher Wiedereingliederungsmaßnahmen für erforderlich. Dies ist in der Praxis durch eine konsequente Umsetzung der auf der Ebene der Bundesarbeitsgemeinschaft Rehabilitation entwickelten „Gemeinsamen Empfehlungen“ durchzusetzen.

\section{Literatur}

${ }^{1}$ Fachverband Sucht e.V. (Hrsg.). Wolfsburger Resolution zur Förderung der Teilhabe am Arbeitsleben von suchtkranken Menschen vom 4.12.2003. Sucht aktuell 2004: 1: $74-75$

2 Bachmeier R et al. In: Fachverband Sucht e. V (Hrsg). Basisdokumentation 2003 - Ausgewählte Daten zur Entwöhnungsbehandlung im Fachverband Sucht e.V. Reihe: Qualitätsförderung in der Entwöhnungsbehandlung. Bd. 12. Bonn: Eigenverlag, 2004

${ }^{3}$ Zobel M, Missel P, Bachmeier M et al. Effektivität der stationären Suchtrehabilitation - FVS - Katamnese des Entlassjahrgangs 2002 von Fachkliniken für Alkohol- und Medikamentenabhängige. Sucht aktuell 2005; 12 (1): $11-20$

${ }^{4}$ Bundesagentur für Arbeit. Leitfaden für die arbeitsamtsärztliche Begutachtung. Missbrauch und Abhängigkeit von Alkohol, Drogen und Medikamenten. Nürnberg, 2004

${ }^{5}$ Deutsche Hauptstelle für Suchtfragen, Fachverband Drogen und Rauschmittel, Fachverband Sucht e.V. Fragebogen für ambulante und stationäre Einrichtungen für Suchtkrankenhilfe zur Integration und Beschäftigung. Bonn, Hamm, März 2005

${ }^{6}$ Bundesversicherungsanstalt für Angestellte. Rundschreiben vom 13.5.2005. „Verbesserung der Eingliederung arbeitsloser Rehabilitanden in das Erwerbsleben nach ihrer Entwöhnungsbehandlung, hier: Kontaktaufnahme mit den Agenturen für Arbeit während der Leistungen zur medizinischen Rehabilitation“. Berlin, 2005 